\title{
Uranium, radium and thorium in soils with high-resolution gamma spectroscopy, MCNP-generated efficiencies, and VRF non-linear full-spectrum nuclide shape fitting
}

\author{
Robert Metzger ${ }^{1, *}$, Kenneth Van Riper ${ }^{2}$, and George Lasche ${ }^{3}$ \\ ${ }^{1}$ Radiation Safety Engineering, Inc, 3245 North Washington Street, Chandler, AZ, USA \\ ${ }^{2}$ White Rock Science, PO Box 4729, Los Alamos, NM, USA \\ ${ }^{3}$ Snakedance Scientific, LLC, 13509 Quaking Aspen Place NE, Albuquerque, NM, USA
}

\begin{abstract}
A new method for analysis of uranium and radium in soils by gamma spectroscopy has been developed using VRF ("Visual RobFit") which, unlike traditional peak-search techniques, fits full-spectrum nuclide shapes with non-linear least-squares minimization of the chi-squared statistic. Gamma efficiency curves were developed for a $500 \mathrm{~mL}$ Marinelli beaker geometry as a function of soil density using MCNP. Collected spectra were then analyzed using the MCNP-generated efficiency curves and VRF to deconvolute the $90 \mathrm{keV}$ peak complex of uranium and obtain ${ }^{238} \mathrm{U}$ and ${ }^{235} \mathrm{U}$ activities. ${ }^{226} \mathrm{Ra}$ activity was determined either from the radon daughters if the equilibrium status is known, or directly from the deconvoluted $186 \mathrm{keV}$ line. ${ }^{228} \mathrm{Ra}$ values were determined from the ${ }^{228} \mathrm{Ac}$ daughter activity. The method was validated by analysis of radium, thorium and uranium soil standards and by inter-comparison with other methods for radium in soils. The method allows for a rapid determination of whether a sample has been impacted by a man-made activity by comparison of the uranium and radium concentrations to those that would be expected from a natural equilibrium state.
\end{abstract}

\section{Introduction}

Rapid determination of uranium and radium concentrations in bulk soil samples is important for waste characterization for naturally-occurring radioactive materials (NORM) rules, determination if soils on a site are impacted by man-made activities, safeguards activities, and accident response. Gamma spectroscopy with high-purity germanium detectors is an attractive approach, however many of the lines needed to determine the uranium and radium concentrations are convoluted and commercially available software packages cannot deconvolute them. Further, there is significant self absorption of photons in the soil sample matrix that affect the efficiency curves needed to quantify results. The changes in photon efficiency vary with the soil density and the sample counting geometry chosen.

To address these deficiencies, detector efficiencies were calculated using MCNP [1] modeling and deconvolution of interfering peaks was accomplished with non-linear fullspectrum nuclide shape fitting using VRF, which is short for "Visual RobFit", and is described in more detail in [2].
These methods were then applied to radium, thorium and uranium soil standards and results were compared to those of commonly-used other methods.

\section{Method}

\subsection{Efficiency curves}

Efficiency curves as a function of soil density for the detector and sample geometry chosen were determined using an MCNP model of the detector and the $500 \mathrm{~mL}$ Marinelli beaker counting geometry used in this work. The model [3] consisted of the crystal, dead layer, detector cover, and the Marinelli beaker. The thickness of the dead layer was estimated by varying the thickness of the layer until the MCNP-generated efficiency values matched the empirically-determined values using a NIST-traceable multiline standard. Once a good agreement was obtained, the matrix in the beaker in the model was changed to a standard soil and MCNP was run to obtain efficiency curves

\footnotetext{
*Corresponding author: rlmetzger@,radsafe.com
} 
for soil densities from 1.1 to $2.0 \mathrm{~g} / \mathrm{cm}^{3}$. The efficiency curves are shown in Figure 1. The values for full-energy peak efficiency that were calculated with MCNP at 60, 88, $122.1,169.5,279.2,391.7,661.7,898,1173.2,1332.5$, and $1836.1 \mathrm{keV}$ were then fitted with a 5-parameter efficiency function using VRF. An example of the efficiency fit to the MCNP values for soil density of $1.3 \mathrm{~g} / \mathrm{cm}^{3}$ that was used for the thoriated soil analysis in this work is shown in Figure 2.

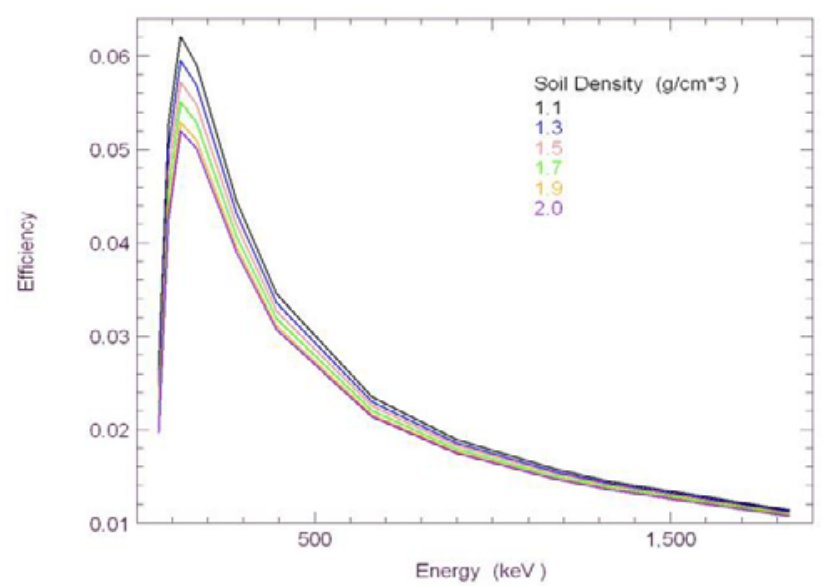

Figure 1. Efficiency curves generated with MCNP for soil in a $500 \mathrm{~mL}$ Marinelli beaker.

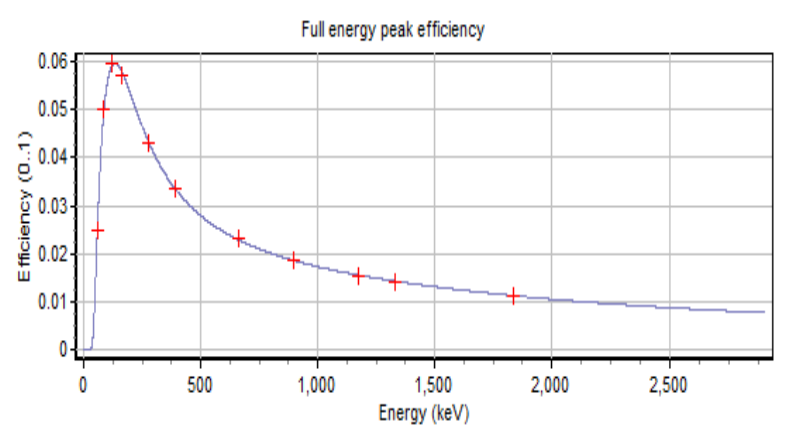

Figure 2. The 5-parameter curve fit (blue) generated by VRF to the efficiency points (red) that were generated with MCNP for a soil density of $1.3 \mathrm{~g} / \mathrm{cm}^{3}$. This efficiency curve was used in the analysis of standard thoriated soil.

\subsection{Spectral analysis}

To deconvolute the overlapped spectra generated by natural sources the VRF gamma spectroscopy software was chosen. This analysis program differs from other commercial software in that it forms, at each of many automated iterations, a spectrum-wide shape for each nuclide and, also at each iteration, it adjusts the activities of each nuclide, as well as user-enabled parameters of energy calibration, attenuation, peak width as a function of energy, coincidence summing, and efficiency until no better fit to the data can be obtained. Other commercial softwares in wide use simply attempt to find each peak in the spectrum and then use tables to identify nuclide activities, with the result that minor peaks that are masked by larger, overlapping peaks cannot be identified. For this reason, VRF is capable of direct measurement of ${ }^{226} \mathrm{Ra}$ (whose peak at $186.2 \mathrm{keV}$ appears to be merged with the peak of ${ }^{235} \mathrm{U}$ at $185.7 \mathrm{keV}$ ), while simple peak-search methods fail. Once the spectrum has been fit, then the spectrum is reassembled and the relative contributions of different lines in overlapped peaks can be seen. Figure 3 shows a segment of the deconvoluted spectrum of a sample of water that was contaminated with depleted uranium. Finally, quantification of the activities in the sample is done using the MCNP-generated efficiency curve that best matches the density of the sample under test.

The resulting method can measure ${ }^{238} \mathrm{U},{ }^{235} \mathrm{U},{ }^{226} \mathrm{Ra}$ and ${ }^{228} \mathrm{Ra}$ in soil samples and can further distinguish man-made contributions of uranium and radium in the soil or water sample to natural concentrations by comparing the measured values to expected equilibrium values based on other isotopes in the chain. The method can evaluate ${ }^{226} \mathrm{Ra}$ by directly measuring the deconvoluted $186.2 \mathrm{keV}$ line, or by the activities of the short-lived radon daughters, if the equilibrium status of the sample is known. Uranium enrichment may also be estimated if the counting statistics for the ${ }^{235} \mathrm{U}$ lines are sufficient. Similarly, ${ }^{232} \mathrm{Th}$ and ${ }^{228} \mathrm{Th}$ concentrations may also be determined when they are present.

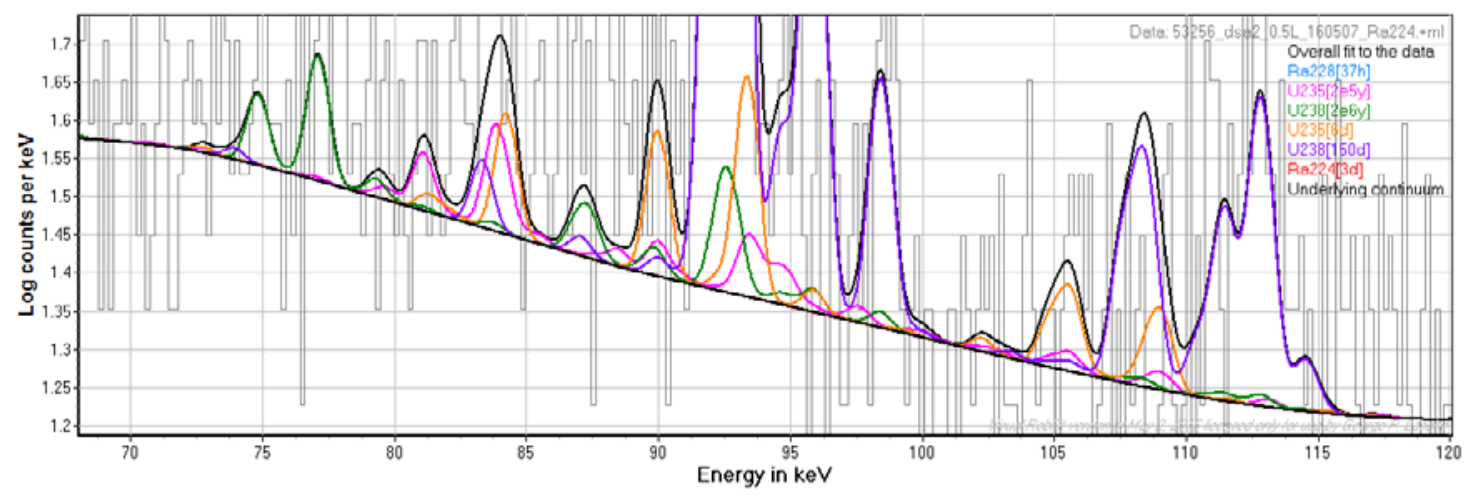

Figure 3. The $90 \mathrm{keV}$ region of a spectrum of water contaminated with depleted uranium and analyzed with VRF. The spectrum has been decomposed into a best fit of full-spectrum shapes of six equilibrium decay chains: geologically aged ${ }^{238} U$ (green) and ${ }^{235} U$ (pink), recently processed ${ }^{238} \mathrm{U}$ (purple) and ${ }^{235} \mathrm{U}$ (orange), and fresh ${ }^{224} \mathrm{Ra}$ (red) and ${ }^{228} \mathrm{Ra}$ (blue). The sum of all fits is shown in black. The decay-chain shapes include decay $\mathrm{x}$-rays of the constituent nuclides in addition to their primary, escape, and coincidence sum peaks. 


\section{Validation}

The method was validated by counting soil standards with known activities of uranium, thorium and radium in them. For the radium isotopes, further validation was made by inter-comparison with other methods for a set of routine samples with low concentrations of radium in them.

\subsection{Uranium}

A uranium standard solution was used to create water and synthetic soil sample standards. The standards were counted on the characterized spectrometer and evaluated as described above. Results are shown in Table 1 below. Results for the ${ }^{238} \mathrm{U}$ were within counting error. The ${ }^{235} \mathrm{U}$ results needed to determine enrichment were within $25 \%$ of the actual values. It should be noted that extended counting times are necessary to obtain an accurate measure of the uranium enrichment at natural and depleted abundances due to the low concentrations of the ${ }^{235} \mathrm{U}$ in environmental samples. At natural abundance, the ${ }^{235} \mathrm{U}$ is $0.71 \%$ of the ${ }^{238} \mathrm{U}$ by mass.

\subsection{Radium}

A radium standard in synthetic soil at $1.3 \mathrm{~g} / \mathrm{cm}^{3}$ was made using a NIST-traceable radium standard, and the standard was counted on the characterized spectrometer. The concentration of ${ }^{226} \mathrm{Ra}$ and ${ }^{228} \mathrm{Ra}$ was determined with the new method and the results are shown in Table 2 below. Results show that the new method was within counting error for the two isotopes. The ${ }^{226} \mathrm{Ra}$ concentration was determined directly from the deconvoluted $186 \mathrm{keV}$ line, while the ${ }^{228} \mathrm{Ra}$ was estimated from the ${ }^{228} \mathrm{Ac} 911 \mathrm{keV}$ line after the ${ }^{228} \mathrm{Ac}$ had been allowed to grow in for 48 hours.
Finally the new method was compared to two existing methods for determining radium in soils for a series of normal soil samples with relatively low natural radium concentrations. The acquired spectra were evaluated using the new method, the Rosson [4] method which uses a direct calibration and two lines each to estimate the ${ }^{226} \mathrm{Ra}$ and ${ }^{228} \mathrm{R}$ a values, and the Canberra Genie [5] software. Since the equilibrium status of the samples were known (full ingrowth), the lines from the ${ }^{214} \mathrm{~Pb}$ and ${ }^{214} \mathrm{Bi}$ were used to determine the ${ }^{226} \mathrm{Ra}$ concentration directly from the Genie analysis software. The MCNP-generated efficiency curves accounted for self-absorption in the different soil densities and were used for all of the methods. Results of this intercomparison are shown in Figures 4 and 5. Results show relatively good agreement across the different methods.

\subsection{Thorium}

A thorium standard in synthetic soil at $1.3 \mathrm{~g} / \mathrm{cm}^{3}$ was made using calibrated thorium ore, and the standard was counted on the characterized spectrometer. The concentration of ${ }^{232} \mathrm{Th}$ and ${ }^{228} \mathrm{Th}$ was determined with the new method and the results are shown in Table 3 and Figure 6 below. Results show that the new method was within counting error for the two primary thorium chain isotopes.

\section{An application}

The new method was used in conjunction with traditional alpha spectroscopy to evaluate a water sample suspected of being impacted by depleted uranium (DU). The water sample was evaluated using SM 7500U-C [6] and the alpha spectrometer spectrum is shown in Figure 7. The spectrum shows a strong disequilibrium between the ${ }^{238} \mathrm{U}$ peak and

Table 1. Uranium in water and uranium in synthetic soil standards by gamma spectroscopy. Only results from VRF are shown because peak-search analyses by Genie and Rosson were unable to deconvolve or resolve critical uranium peaks at this low level of activity.

\begin{tabular}{|c|c|c|c|}
\hline & Actual (Bq/g) & VRF (Bq/g) & \% Error \\
\hline${ }^{238} \mathrm{U}$ in $\mathrm{H}_{2} \mathrm{O}$ & 0.0069 & 0.0061 & 9.7 \\
\hline${ }^{235} \mathrm{U}$ in $\mathrm{H}_{2} \mathrm{O}$ & 0.00031 & 0.00044 & 26 \\
\hline${ }^{238} \mathrm{U}$ in Soil & 1.07 & 1.08 & 0.59 \\
\hline${ }^{235} \mathrm{U}$ in Soil & 0.054 & 0.050 & 8.25 \\
\hline
\end{tabular}

Table 2. Radium by gamma spectroscopy. Radium standard in synthetic soil at $1.3 \mathrm{~g} / \mathrm{cm}^{3}$.

\begin{tabular}{|c|c|c|c|c|c|c|c|}
\hline & Actual (Bq/g) & Genie (Bq/g) & \% Error & Rosson (Bq/g) & \% Error & VRF (Bq/g) & \% Error \\
\hline${ }^{226} \mathrm{Ra}$ & 0.019 & 0.020 & 4.6 & 0.021 & 10.5 & 0.019 & 0.03 \\
\hline${ }^{228} \mathrm{Ra}$ & 0.014 & 0.013 & 2.7 & 0.013 & 8.1 & 0.0145 & 3.45 \\
\hline
\end{tabular}


the ${ }^{234} \mathrm{U}$ peak and a much lower enrichment $(0.2 \%)$ than would be expected in a natural source $(0.71 \%)$. While $234 / 238$ disequilibrium is not uncommon in natural sources, it is normally the slightly more soluble ${ }^{234} U$ that has the higher activity. The strong disequilibrium in favor of the ${ }^{238} \mathrm{U}$ is characteristic of depleted uranium, a waste product from the enrichment process that has excellent shielding properties, and is also used as an anti-tank projectile.

The sample was also evaluated using the new method and the deconvoluted spectrum of the crucial $90 \mathrm{keV}$ peak complex of uranium was shown earlier in Figure 3. The enrichment estimate by the gamma spectrometer method is shown in Table 4, and also shows that the uranium in the sample is primarily depleted uranium. The other gamma spectroscopy methods were unable to determine uranium activity or enrichment as they cannot deconvolute the heavily overlapped spectrum.

This method was also able to estimate the extent to which the sample was impacted by the DU. Natural uranium, of course, was also present in the sample. The other natural chain isotopes in the sample were used to estimate expected values of natural uranium that should have been present, and the DU to natural ratio was found to be 49:1, clearly indicating the sample was primarily DU.

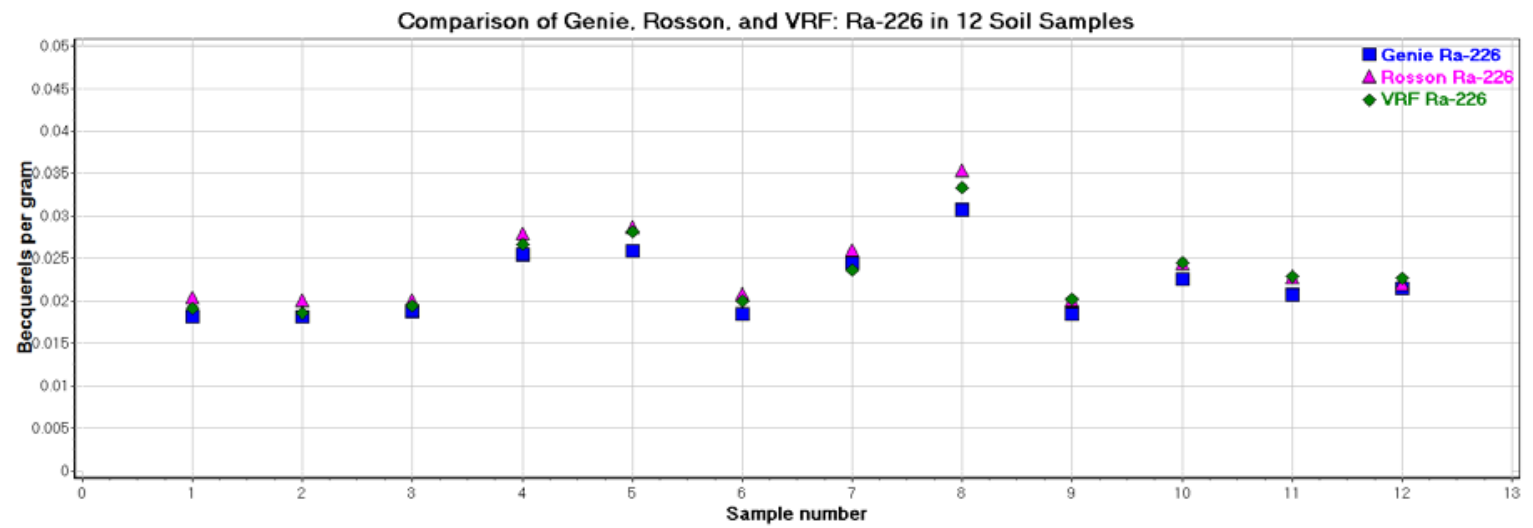

Figure 4. Comparison of analysis results from Genie, the method of Rosson, and VRF of specific activities of ${ }^{226}$ Ra in 12 soil samples with densities of $1.3 \mathrm{~g} / \mathrm{cm}^{3}$ in $500 \mathrm{~mL}$ Marinelli beakers. The efficiency curve was modeled with MCNP.

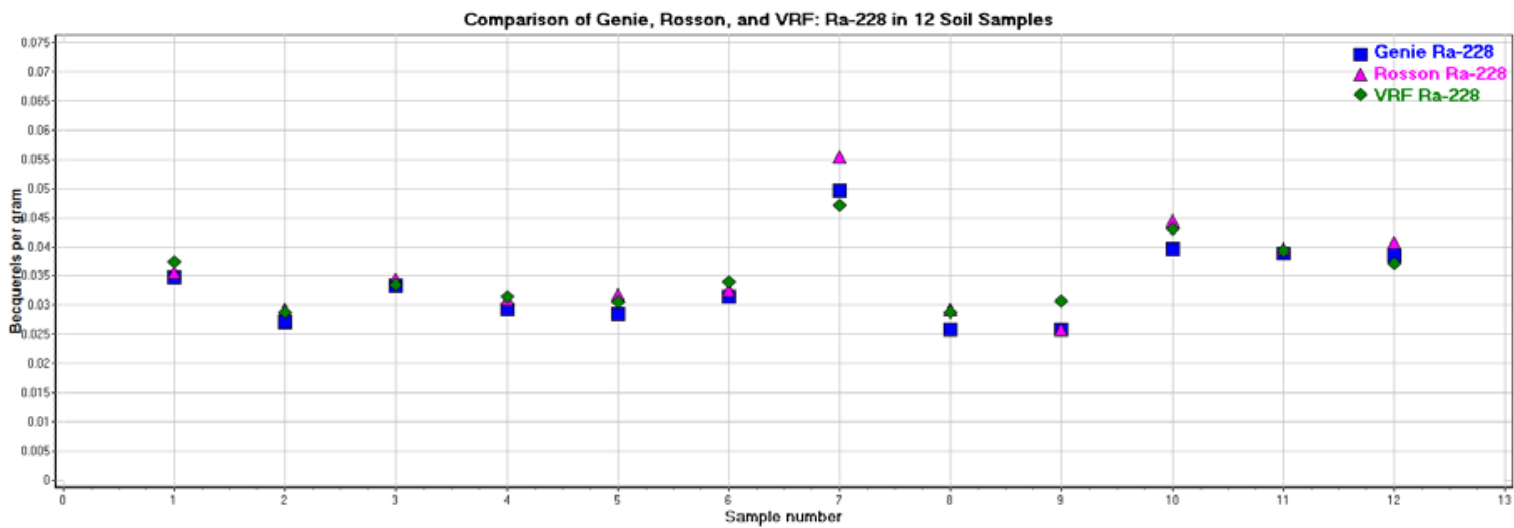

Figure 5. Comparison of analysis results from Genie, the method of Rosson, and VRF of specific activities of ${ }^{228}$ Ra in 12 soil samples with densities of $1.3 \mathrm{~g} / \mathrm{cm}^{3}$ in $500 \mathrm{~mL}$ Marinelli beakers. The efficiency curve was modeled with MCNP.

Table 3. Thorium in synthetic soil standards.

\begin{tabular}{|c|c|c|c|}
\hline & Actual (Bq/g) & VRF (Bq/g) & \% Error \\
\hline${ }^{232} \mathrm{Th}$ & 1.58 & 1.64 & 3.42 \\
\hline${ }^{228} \mathrm{Th}$ & 1.58 & 1.64 & 3.42 \\
\hline
\end{tabular}




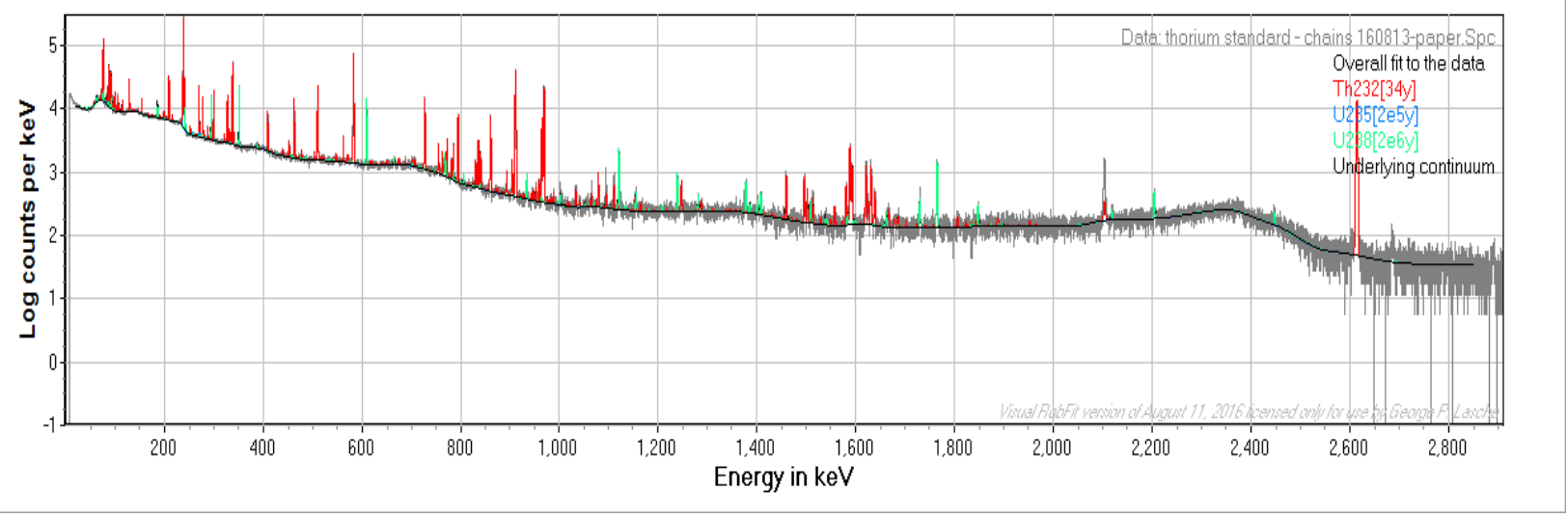

Figure 6. Graphical results of analysis with VRF of thoriated standard soil. In this case VRF was used to fit entire equilibrium decay chains as one emitting source. The equilibrium decay chain of ${ }^{232} \mathrm{Th}$ is shown in red and is evidenced primarily by peaks from ${ }^{228} \mathrm{Ac}$, ${ }^{212} \mathrm{~Pb}$, and ${ }^{208} \mathrm{Tl}$. The equilibrium decay chain of ${ }^{238} \mathrm{U}$ is shown in green and is evidenced primarily by peaks from ${ }^{214} \mathrm{Bi},{ }^{214} \mathrm{~Pb}$, ${ }^{226} \mathrm{Ra}$, and ${ }^{234 \mathrm{~m}} \mathrm{~Pa}$.

Table 4. Analysis of impacted water sample in good agreement assessing the uranium as DU. Uranium enrichment by alpha spec and VRF (natural abundance of uranium is $0.71 \%$ ).

\begin{tabular}{|c|c|}
\hline Alpha spec (\%) & VRF (\%) \\
\hline $0.2+/-0.001$ & $0.3+/-0.084$ \\
\hline
\end{tabular}

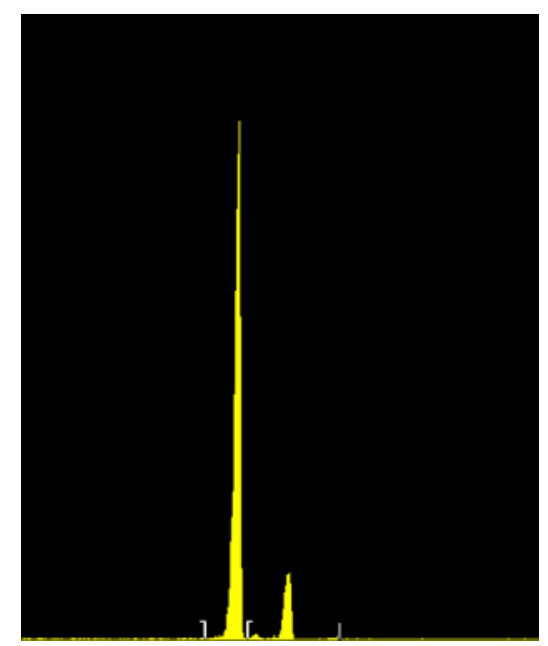

Figure 7. Alpha spectrum of impacted uranium in water sample, showing a strong disequilibrium between the ${ }^{238} \mathrm{U}$ peak and the ${ }^{234} \mathrm{U}$ peak and a much lower enrichment $(0.2 \%)$ than would be expected in a natural source $(0.71 \%)$.

\section{Conclusion}

A new method has been developed that is especially useful for determining uranium and radium concentrations in soil or water samples by gamma spectroscopy using conventional P- or N-type HPGe spectrometers. The method is less expensive and requires less time than traditional methods that solubilize the soil sample through acid leaching or various fusion methods, and which then perform radiochemical separations and alpha spectroscopy. The new spectroscopic analysis techniques of VRF can directly measure ${ }^{226} \mathrm{Ra}$ concentrations without the usual ingrowth time for its daughter nuclides. The method can determine uranium enrichment and whether soils are impacted by some man-made activity or are natural. 


\section{References}

1. T. Goorley, M. James, T. Booth, F. Brown, J. Bull, L. J. Cox, J. Durkee, J. Elson, M. Fensin, R. A. Forster, J. Hendricks, H. G. Hughes, R. Johns, B. Kiedrowski, R. Martz, S. Mashnik, G. McKinney, D. Pelowitz, R. Prael, J. Sweezy, L. Waters, T. Wilcox, T. Zukaitis, "Initial MCNP6 Release Overview", Nuclear Technology, 180, pp.298-315,(2012)

2. G.P. Lasche, R.L. Coldwell, R.L. Metzger, "VRF nuclear spectral analysis with non-linear wholespectrum nuclide shape fitting", to be published in the proceedings of the ICRS-13 \& RPSD-2016, Paris, France, Oct 3-6, 2016.
3. R. Metzger, K. Van Riper, "A Monte Carlo Approach to Food Density Corrections in Gamma Spectroscopy", Journal of Radioanalytical and Nuclear Chemistry, DOI: 10.1007 (2015)

4. B. Kahn, R. Rosson, J. Cantrell, "Analysis of Ra-228 and Ra-226 In Public Water Supplies by a Gamma Ray Spectrometer," Health Physics, 59, 125-131, (July 1990)

5. Genie 2000 Operations Manual (3.2 ed.), Canberra Industries. (2009)

6. L. Clesceri, A. Greenberg,, A. Eaton, Standard Methods for the Examination of Water and Wastewater (20th ed., pp. 7-47 - 7-51). Washington: APHA American Public Health Association (1998) 